\begin{tabular}{|c|l|}
\hline Title & Skewed soldier sex ratio in termites: testing the size threshold hy pothesis \\
\hline Author(s) & Bourguignon, Thomas; Hay ashi, Y oshinobu; Miura, Toru \\
\hline Citation & $\begin{array}{l}\text { Insectes Sociaux, 59(4), 557-563 } \\
\text { https://doi.org/10.1007/300040-012-0252-8 }\end{array}$ \\
\hline Issue Date & 2012-11 \\
\hline Doc URL & http://hdl.handle.net/2115/50723 \\
\hline Rights & The final publication is available at www.springerlink.com \\
\hline Type & article (author version) \\
\hline File Information & IS59-4_557-563.pdf \\
\hline
\end{tabular}

Instructions for use 


\section{Skewed soldier sex ratio in termites: testing the size-threshold hypothesis}

Running head: Soldier sex ratio in termites

Thomas Bourguignon ${ }^{1}$ Yoshinobu Hayashi ${ }^{1}$ and Toru Miura ${ }^{1}$

${ }^{1}$ Graduate School of Environmental Science, Hokkaido University, Sapporo 060-0810, Japan

Correspondence: Thomas Bourguignon, Graduate School of Environmental Science, Hokkaido University, Sapporo 060-0810, Japan.

Email: thomas_bourguignon@ees.hokudai.ac.jp

Number of words: 4465 
Abstract Social insect colonies contain multiple phenotypes, i.e. castes, and this caste polyphenism is often linked to sexual dimorphism. Unlike social hymenopterans, both termite sexes are diploid and contribute to helper-type tasks within the colony. Nonetheless, a biased sex ratio is frequently observed in termites, especially in soldiers. To explain this bias in soldier sex ratio, Matsuura (2006) postulated the existence of a size threshold for workers molting into soldiers. Under the influence of sexual size dimorphism (SSD), such a threshold was considered to indirectly favor one sex. We conducted a literature survey of sex ratio among termite soldiers and tested this size-threshold hypothesis using data for 67 termite species from a variety of termite lineages. We demonstrated the existence of a size threshold for individuals molting into soldiers, resulting in the acquisition of soldiers of only one sex in species exhibiting strong SSD. In species exhibiting weak SSD, the size threshold skews the sex ratio of soldiers, but does not necessarily cause the loss of one sex. Finally, we observed a prevalence of single-sex soldiers in the Termitidae, regardless of SSD, suggesting that the ancestral developmental mechanisms that constrain soldier differentiation from one sex are maintained in certain extant species.

Keywords Caste polymorphism, Isoptera, termite evolution, phylogeny, sexual dimorphism 


\section{Introduction}

Social insects have achieved the highest level of social organization, with some individuals sacrificing their own reproductive fitness to help their parents or other genetically related individuals to reproduce (Hamilton 1964a, b). In social hymenopterans, haploid males are produced from unfertilized eggs while diploid females are produced from fertilized eggs. This haplodiploid sex-determination system creates asymmetric relatedness between sexes, which is one of the factors that contributed to the establishment of strictly all-female helpers (Trivers and Hare 1976; Howard and Thorne 2011). An interesting analogy to social hymenopteran haplodiploidy exists in termites and implies the existence of some sort of sex chromosome linkage during male meiosis in some species; however, its implication in the production of unequal sex ratios is dubious (Thorne 1997; Roisin 2001). In termites, both sexes are diploid and the sex of helpers (workers and soldiers) is not influenced by relatedness asymmetries. In the basal termite families Kalotermitidae and Termopsidae, workers (or pseudergates) and soldiers have sex ratios that are either equal or only slightly biased (Roisin 2000). However, this ancestral condition has evolved into strongly biased sex ratio in many Termitidae and to a lesser extent in the Hodotermitidae and Rhinotermitidae, with many species having soldiers of only one sex and workers with both sexes (Roisin 2000).

The soldier caste in termites is remarkable for its huge morphological diversity, reflecting adaptations to optimize colony defense (Deligne et al. 1981). It is widely considered that the soldier caste arose only once in the common ancestor of termites (Roisin 2000). Soldiers are the only caste that is always permanently sterile, although some soldierlike reproductives occasionally occur in Zootermopsis species (Myles 1986; Thorne 1997; Roisin 2000). Noirot (1951) was the first to report a skewed sex ratio in soldiers, and this was followed shortly by the discovery of the phenomenon in a further 30 species within the 
Termitidae (Noirot 1955). Since then, sex ratios have been described in another 30 species, providing researchers with a comprehensive understanding of sex ratios in termites (Table S1). However, while sex specialization in termite soldiers is a well described phenomenon, we are still very far from thoroughly understanding its origins.

One proximate hypothesis for the origin of single-sex soldiers is the existence of a sexlinked, genetic, caste determination system (Lo et al. 2009). However, on rare occasions, or in instances where termites have been reared artificially under abnormal caste compositions, species with single-sex soldiers, e.g. Acanthotermes acanthothorax, Microcerotermes parvus and Nasutitermes arborum (Macrotermitinae, Termitinae and Nasutitermitinae), can produce soldiers of the opposite sex (Noirot 1955). This finding demonstrates that both sexes possess the genetic architecture that is required to develop into soldiers. The genetic hypothesis may therefore either be invalid, or imply sexual differences in sensitivity to soldier differentiation signals.

A non-exclusive hypothesis for the ultimate factors driving single-sex soldiers was recently proposed by Matsuura (2006). He noted that Reticulitermes (Rhinotermitidae), as numerous other termite species, relies upon a static warfare defensive strategy, in which powerful soldiers guard the nest entrance and plug the galleries with their heads (Deligne and Pasteels 1982; Matsuura 2002). As soldiers must be sufficiently large to fit the galleries and efficiently plug them, there should be a size threshold below which workers are unable to molt into soldiers. Thus, in the case of sexual size dimorphism (SSD) in termites, the sex ratio of soldiers should be biased toward the larger sex, while in absence of SSD, Matsuura (2006) suggested that both sexes should be equally represented. Conversely, in the case of mobile warfare defensive strategy, numerous soldiers are produced, which must be small enough to be sustained by the colony. In this case, Matsuura (2006) suggested that the smaller sex should be indirectly favored. The hypothesis works well in four Reticulitermes species, but 
not completely in Cryptotermes secundus (Kalotermitidae) whose soldier sex ratio is biased while SSD is absent (Matsuura 2006; Muller and Korb 2008).

We conducted a literature survey on soldier sex ratios and SSD in 67 termite species from around the world. Our primary goal was to elucidate the origin of the biased soldier sex ratio and to map its evolution along the termite phylogenetic tree. According to the size threshold hypothesis (Matsuura 2006), biased soldier sex ratio is a byproduct from the selection for soldiers of the optimal size, maximizing their defensive abilities. Selection on soldier size was therefore suggested to be a key factor regulating soldier sex ratio (Matsuura 2006). Here, we carried out a meta-analysis and tested the following hypotheses:

1. Selection favors appropriate sizes of soldiers for effective defense, biasing the sex ratios in presence of SSD. We therefore hypothesized that SSD is correlated with skewed sex ratio of soldiers.

2. Another factor that might affect soldier sex ratios is species phylogenetic history. Here, we hypothesized that the preponderance of single-sex soldiers in Termitidae is partly due to inheritance from their ancestor which had strong SSD and single-sex soldiers.

\section{Material and Methods}

\section{Literature survey}

Most of the data on soldier morphometry and sex ratios were extracted from studies related to termite caste systems. We conducted a literature search using the keywords "termite caste system" in the ISI Web of Knowledge, or by referring to literature cited by Roisin (2000), who reviewed the current status of the data on termite caste systems. Altogether, our dataset includes the outputs of 27 papers (Table S1) 


\section{Data analyses}

In the few species with single-sex workers, the soldiers are of the same sex as the workers. In such cases, the biased sex ratio of single-sex soldiers is an indirect consequence of single-sex workers. We therefore restricted our analysis to termite species with workers of both sexes whose biased soldier sex ratio results from direct selection of the soldier sex. Species with dimorphic soldier caste were treated as a single entry.

Given that related species share a common history, they are not statistically independent from each other (Pagel 1994). As a result, classical statistics cannot be applied to study the evolution of traits among related species. We therefore subjected the data to Pagel's discrete correlation analysis which considers the underlying phylogeny to correct for the nonindependence of data points (Pagel 1994). The phylogenies were based on cytochrome oxidase II (COII), 12SrDNA and 28SrDNA sequences of 245 species that had been deposited in GenBank, primarily by Inward et al. (2007). Since no sequence data were available for Labidotermes celisi, we used sequences of Speculitermes sp., which also belongs to the Apicotermitinae (Inward et al. 2007). Sequences were aligned using the Mega_5.0 package (Tamura et al. 2011), using the Muscle algorithm and then concatenated with Mesquite (Maddison and Maddison 2010). Sequence alignments were visually checked and ambiguous alignments were ignored to produce a final data matrix with $1688 \mathrm{bp}$. We employed the maximum likelihood method, and a set of 100 trees computed using Bayesian method. The maximum likelihood method was implemented in DNAml in the Phylip-3.69 suite of programs for Mac OS X to generate a phylogeny under default setting: assuming that each site in the sequence evolves independently, that different lineages evolve independently and that the rate of variation was constant among sites. We also used a single category of sites (Felsenstein 2005). The Bayesian trees were computed using Markov Chain Monte Carlo 
algorithm, following a GTR model with gamma-distributed rate variation across sites and implemented in Mr Bayes version 3.1.1 (Ronquist and Huelsenbeck 2003). The run length was one million generations, from which we sampled one tree every 5000 generations started from the 505000th generation. From the trees we obtained, we pruned all the leaves for which we did not have data.

A convenient approach to test the size-threshold hypothesis is to consider the most extreme cases of skewed soldier sex ratios, namely, species with single-sex soldiers. Using a binary character (single-sex vs both sexes soldiers), we compared species having strong SSD with species having low or missing SSD. We tested for a correlation between single-sex soldiers and strong SSD, using a Pagel's discrete correlation test with Mesquite (Maddison and Maddison 2010). Specifically, we tested the correlation between two sets of discrete values: low or no SSD and strong SSD versus single and both-sex soldiers. The low or no SSD category included species with head width differing by less than $3 \%$ between sexes, or with histogram of head width clearly showing an unimodal distribution; while the strong SSD category included species with head width differing by at least $10 \%$ between sexes. No species exhibiting moderate SSD (i.e. between strong and weak SSD) have been reported. The p-value was estimated using 5000 simulations with the maximum likelihood tree and using 1000 simulations with 10 Bayesian trees (those trees were obtained after 550000, 600000 ... 1000000 generations) Using the same analyses and number of simulations, we also tested the relevance of the size-threshold hypothesis for the species with low and missing SSD. More precisely, we tested for a correlation between SSD (low and missing) and biased sex ratio (biased and unbiased).

To better understand the evolution of SSD and single-sex soldiers, we reconstructed their ancestral states on all phylogenetic trees of termites using Mesquite (Maddison and Maddison 2010). For this purpose, we used likelihood analyses using AsymmMk model and 
the Mk1 model, as well as parsimony analysis. The Mk1 model has a single parameter, the rate of change, and therefore supposes that any particular change is equally probable; while the AsymmMk model possesses two parameters, one for the forward rate of change and one for the backward rate of change, allowing a bias in gain versus loss. The parsimony method finds the ancestral state that minimizes the number of changes along the phylogenetic tree.

\section{Results and discussion}

Sex ratio in termites

While the helpers (workers and soldiers) of lower termites generally belong to both sexes (Roisin 2000), there are exceptions: (1) in Hodotermes mossambicus (Hodotermitidae) and Reticulitermes virginicus (Rhinotermitidae), workers of both species consist of both sexes, but the soldiers are all male in H. mossambicus (Luamba 1980) and all female in R. virginicus (Matsuura 2006); (2) in Glossotermes oculatus (Serritermitidae), all helpers are male, except for shortly before the swarming period when female helpers appear and rapidly molt into alates (Bourguignon et al. 2009). Conversely, in the Rhinotermitinae and Anacanthotermes ahngerianus, workers and soldiers are all female and male, respectively (Table S1) (Renoux 1976; Mednikova 1977; Roisin 1988a).

Helper sex ratios in the Termitidae differ markedly from those observed in the lower termites. The worker and the soldier sex ratio in lower termites are often balanced, or at least, both sexes are represented; however, this is rarely the case in the Termitidae in which the single-sex soldier condition is common. A few exceptions have also been reported in the genus Amitermes (Termitinae) and in the two Neotropical, soil-feeding, nasute genera, Coatitermes and Subulitermes (Nasutitermitinae) whose soldiers consist of both sexes (Noirot 
1955; Roisin 1996). As in lower termites, there are species whose helpers are all male, e.g. Cornitermes (Syntermitinae) and Constrictotermes (Nasutitermitinae) (Roisin 1992; Moura et al. 2011) (Table S1).

Hypothesis 1. Sexual size dimorphism is correlated with skewed sex ratio of soldiers Altogether, we recorded 31 species that exhibited strong SSD and had soldiers of a single sex, while only one had strong SSD and soldiers of both sexes. Of the 29 species exhibiting either weak or no SSD, 14 have single-sex soldiers and 15 have soldiers of both sexes (Fig. 1). A significant correlation was observed between SSD and soldier sex (Likelihood tree: Pagel's discrete correlation: p-value from 5000 simulations $<0.001$; Bayesian trees: Pagel's discrete correlation: $\mathrm{p}$-values from 1000 simulations for 10 trees $\leq 0.001)$, confirming that single-sex soldiers occur when SSD is strong. Conversely, in species with low or no SSD, soldiers of both sexes are more commonly encountered. The size threshold is likely to be more apparent in species exhibiting a strong SSD, as producing soldiers of an inadequate size might compromise their defensive abilities. Thus, in species exhibiting strong SSD, selection for soldiers of the appropriate size will push soldiers toward being of a single sex. As postulated by Matsuura (2006), the indirectly favored sex is not necessarily the larger, but can also be the smaller, e.g. in Nasutitermes. This is likely the consequence of soldier defensive strategy types, namely static and mobile warfare, which require large and small soldiers, respectively (Deligne and Pasteels 1982; Noirot and Darlington 2000). A static warfare strategy consists of producing a small number of soldiers, which are large enough to plug the gallery entrances. Conversely, in mobile warfare species, which produce numerous soldiers that are capable of surging rapidly to outnumber intruders at the point of attack, soldiers are small so that the colony can sustain many of them. Our results indicate that the size threshold hypothesis does not only apply to species with phragmotic soldiers, plugging the nest galleries with their head, 
but also to mobile warfare species in which soldiers must be small enough. Here, we postulate that the selection of large and powerful soldiers might be sufficient for the establishment of a size threshold in static warfare species and that soldiers do not necessarily need to be phragmotic.

Matsuura (2006) initially postulated that biased soldier sex ratios occur in species that exhibit weak SSD. To test this hypothesis, we examined whether the presence of skewed soldier sex ratio is more common in species with weak SSD than in species without SSD. We found that the sex ratio tends to be more often biased in species with weak SSD (Likelihood tree: Pagel's discrete correlation: p-value from 5000 simulations $=0.029$; Bayesian trees: Pagel's discrete correlation: $p$-values from 1000 simulations for 10 trees: $0.025<p<0.036$ ), which corroborates the size-threshold hypothesis. However, unlike species exhibiting strong SSD, numerous species with no or low SSD retain soldiers of both sexes. The finding that some species with no SSD possess biased sex ratios (Muller and Korb 2008) or single-sex soldiers (Noirot 1955) indicates that in the absence of SSD, sex ratio is not necessarily egalitarian. By contrast, soldier sex ratio is always biased in presence of SSD.

Hypothesis 2. Species phylogenetic history influences soldier sex ratio

The prevalence of single-sex soldiers between the Termitidae and lower termites differs when SSD is weak or absent, with members of the Termitidae generally having single-sex soldiers while the lower termites generally retain both sexes (Fisher exact test: $\mathrm{p}=0.003$ ) (Fig. 1). We hypothesize that this major difference between the Termitidae and the lower termites mainly results from phylogenetic constraints. By reconstructing the ancestral state of the Termitidae, we found that their most recent ancestor most likely had single-sex soldiers, possibly coupled with a strong SSD, which was then subsequently lost in some lineages of the Termitinae and 
Nasutitermitinae (Figs 2, 3). The soldiers of both sexes were restored at least twice independently in three genera lacking SSD (Figs 2, 3). This scenario is strongly supported by all ancestral state reconstructions performed on the maximum likelihood tree (Fig. 2), but not on the Bayesian trees, on which the Termitidae ancestor SSD could not be precisely assessed (Fig. 3). Therefore, although realistic, our hypothesis requires further testing as new molecular phylogenies appear. Additionally, it is not clear why soldiers of both sexes were not recovered more often in members of the Termitidae lacking SSD. It is possible that the production of soldiers of both sexes does not confer any advantage on the colony compared to making single-sex soldiers. In addition, in the absence of new selective pressures favoring the development of a mixed-sex soldier caste, re-establishing the second sex in the soldier caste is likely to be a very slow process. Conversely, having a strong SSD is uncommon in the lower termites whose most recent common ancestor had soldiers of both sexes with weak or no SSD (Fig. 2, 3). Therefore, in lower termites, no selective pressures favored the acquisition of single-sex soldiers and both sexes have generally been retained. Altogether, our results suggest that the prevalence of single-sex soldiers in Termitidae might come from their common ancestor, but additional tests are required to validate this hypothesis.

\section{Perspectives}

Our results support the size threshold hypothesis, which postulates that the biased sex ratio of soldiers is indirectly influenced by SSD. However, the factor(s) responsible for the SSD in the neuter line of many termite species are unknown and deserve to be investigated. The sizethreshold hypothesis could also be investigated using experimental bioassays designed to test the relationship between soldier defensive abilities and body size in a static warfare species. In such a scenario, large soldiers are expected to exhibit higher defensive abilities; if 
confirmed, then this would provide additional support for the size-threshold hypothesis. Finally, some developmental mechanisms must cause single-sex soldier. One possibility that deserves to be investigated is sexual differences in physiological states, such as a recurrently high juvenile hormone titer in helpers of one sex. Juvenile hormone titer regulates soldier differentiation in termites (Liu et al. 2005; Mao et al. 2005; Cornette et al. 2008) and therefore represents a good proximate candidate for the selection of one sex.

Acknowledgments We are grateful to the Japan Society for Promotion of Science, for financial support through a postdoctoral fellowship to TB (No. 22-00393) and YH (No. 212869). This work was also supported by Grants-in-Aid for Young Scientists (No. 21677001) from the Ministry of Education, Culture, Sports, Science and Technology of Japan.

\section{References}

Bourguignon T., Šobotnìk J., Hanus R. and Roisin Y. 2009. Developmental pathways of Glossotermes oculatus (Isoptera, Serritermitidae): at the cross-roads of worker caste evolution in termites. Evol. Dev. 11: 659-668

Buchli H.R. 1958. L'origine des castes et les potentialités ontogénétiques des termites européens du genre Reticulitermes Holmgren. Ann. Sci. Nat. Zool. (11 ${ }^{e}$ sér.) 20: 263-429

Cornette R., Gotoh H., Koshikawa S. and Miura T. 2008. Juvenile hormone titers and caste differentiation in the damp-wood termite Hodotermopsis sjostedti (Isoptera, Termopsidae). J. Insect. Physiol. 54: 922-930

Deligne J. and Pasteels J.M. 1969. Morphologie, développement et affinités de Labidotermes celisi gen. nov., sp. n. (Isoptères Amitermitinae du Kivu). Rev. Zool. Bot. Afr. 79: 145- 
164

Deligne J. and Pasteels J.M. 1982. Nest structure and soldier defense: an integrated strategy in termites. In: The biology of social insects. Proceedings of the Ninth Congress of the International Union for the Study of Social Insects (Breed M.D., Michener C.D. and Evans H.E., Eds), Boulder, Colorado: Westview Press. pp 288-289

Deligne J., Quennedey A. and Blum M.S. 1981. The enemies and defense mechanisms of termites. In: Social Insects, Vol. II (Hermann H.R. Ed.), New York, NY: Academic Press. pp $1-76$

Felsenstein J. 2005. PHYLIP (Phylogeny Inference Package) version 3.6. Distributed by the author. Department of Genome Sciences, University of Washington, Seattle

Hamilton W.D. 1964a. The genetical evolution of social behaviour. I. J. Theor. Biol. 7: 1-16

Hamilton W.D. 1964b. The genetical evolution of social behaviour. II. J. Theor. Biol. 7: 1752

Henderson G. and Rao K.S.P. 1993. Sexual dimorphism in soldiers of Formosan subterranean termites (Isoptera: Rhinotermitidae). Sociobiology 21: 341-345

Hojo M., Koshikawa S., Matsumoto T. and Miura T. 2004. Developmental pathways and plasticity of neuter castes in Nasutitermes takasagoensis (Isoptera: Termitidae). Sociobiology 44: 433-441

Howard K.J. and Thorne B.L. 2011. Eusocial evolution in termites and Hymenoptera. In: Biology of termites: A modern synthesis (Bignell D.E., Roisin Y. and Lo N. Eds), Dordrecht: Springer. pp 97-132

Inward D.J.G., Vogler A.P. and Eggleton P. 2007. A comprehensive phylogenetic analysis of termites (Isoptera) illuminates key aspects of their evolutionary biology. Mol. Phylogenet. Evol. 44: 953-967 
Liu Y.X., Henderson G., Mao L.X. and Laine R.A. 2005. Seasonal variation of juvenile hormone titers of the Formosan subterranean termite, Coptotermes formosanus (Rhinotermitidae). Environ. Entomol. 34: 557-562

Lo N., Hayashi Y. and Kitade O. 2009. Should environmental caste determination be assumed for termites? Am. Nat. 173: 848-853

Luamba J.L.N. 1980. Recherches sur le polymorphisme et aperçu sur l'influence de l'analogue de l'hormone juvénile sur le développement d'un termite, Hodotermes mossambicus (Isoptera, Hodotermitidae). Biol. Ecol. médit. 7: 169-171

Maddison W.P. and Maddison D.R. 2010. Mesquite: a modular system for evolutionary analysis. Version $2.73 \mathrm{http}: / /$ mesquiteproject.org

Mao L.X., Henderson G., Liu Y.X. and Laine R. A. 2005. Formosan subterranean termite (Isoptera: Rhinotermitidae) soldiers regulate juvenile hormone levels and caste differentiation in workers. Ann. Entomol. Soc. Am. 98: 340-345

Matsuura K. 2002. Colony-level stabilization of soldier head width for head-plug defense in the termite Reticulitermes speratus (Isoptera: Rhinotermitidae). Behav. Ecol. Sociobiol. 51: $172-179$

Matsuura K. 2006. A novel hypothesis for the origin of the sexual division of labor in termites: which sex should be soldiers? Evol. Ecol. 20: $565-574$

Mednikova T.K. 1977. Caste differentiation in the termite Anacanthotermes ahngerianus Jacobson (Isoptera, Hodotermitidae). In: Proceedings of the Eighth International Congress of the International Union for the Study of Social Insects (Velthuis H.H.W. and Wiebes J.T. Eds), Wageningen, the Netherlands: Centre for Agricultural Publishing and Documentation. pp 118-120

Miura T., Hirono Y., Machida M., Kitade O. and Matsumoto T. 2000. Caste developmental system of the Japanese damp-wood termite Hodotermopsis japonica (Isoptera: 
Termopsidae). Ecol. Res. 15: 83-92

Miura T., Roisin Y. and Matsumoto T. 1998. Developmental pathways and polyethism of neuter castes in the processional nasute termite Hospitalitermes medioflavus (Isoptera: Termitidae). Zool. Sci. 15: 843-848

Moura F.M.S., Vasconcellos A., Silva N.B. and Bandeira A.G. 2011. Caste development systems of the Neotropical termite Constrictotermes cyphergaster (Isoptera, Termitidae). Insect. Soc. 58: 169-175

Muller H. and Korb J. 2008. Male or female soldiers? An evaluation of several factors which may influence soldier sex ratio in lower termites. Insect. Soc. 55: 213-219

Myles T.G. 1986. Reproductive soldiers in the Termopsidae (Isoptera). Pan-Pac. Entomol. 62: $293-299$

Myles T.G. and Chang F. 1984. The caste system and caste mechanisms of Neotermes connexus (Isoptera: Kalotermitidae). Sociobiology 9: 163-319

Neoh K.B. and Lee C.Y. 2009. Developmental stages and castes of two sympatric subterranean termites Macrotermes gilvus and Macrotermes carbonarius (Blattodea: Termitidae). Ann. Entomol. Soc. Amer. 102: 1091-1098

Noirot C. 1951. Le développement des neutres chez les termites supérieurs (Termitidae). IV Le sexe des diverses catégories de neutres. C. R. Acad. Sci. 233: 447-449

Noirot C. 1955. Recherches sur le polymorphisme des termites supérieurs (Termitidae). Ann. Sci. Nat. Zool. (11 sér.) 17: 399-595

Noirot C. 1969. Formation of castes in higher termites. In: Biology of termites. Vol. 1 (Krishna K. and Weesner F.M. Eds), London: Academic Press. pp 311-350

Noirot, C. and Darlington J.P.E.C. 2000. Termite nests: architecture, regulation and defense. In: Termites: Evolution, Sociality, Symbioses, Ecology (Abe, T., Bignell, D.E., Higashi, M. Eds), the Netherlands, Dordrecht: Kluwer Academic Publishers. pp 121-139 
Okot-Kotber B.M. 1985. Mechanisms of caste determination in a higher termite, Macrotermes michaelseni (Isoptera, Macrotermitinae). Curr. Themes Trop. Sci. 3: 267306

Pagel M. 1994. Detecting correlated evolution on phylogenies: a general method for the comparative analysis of discrete characters. Proc. R. Soc. Lond. (Biol.) 255: 37-45

Parmentier D. and Roisin Y. 2003. Caste morphology and development in Termitogeton nr. planus (Insecta, Isoptera, Rhinotermitidae). J. Morphol. 255: 69-79

Renoux J. 1976. Le polymorphisme de Schedorhinotermes lamanianus (Sjöstedt) (IsopteraRhinotermitidae). Essai d'interprétation. Insect. Soc. 23: 279-494

Roisin Y. 1988a. The caste system of Parrhinotermes browni (Isoptera: Rhinotermitidae). Sociobiology 14: 21-28

Roisin Y. 1988b. Morphology, development and evolutionary significance of the working stages in the caste system of Prorhinotermes (Insecta, Isoptera). Zoomorphology 107: $339-347$

Roisin Y. 1992. Development of nonreproductive castes in the Neotropical termite genera Cornitermes, Embiratermes and Rhynchotermes (Isoptera: Nasutitermitinae). Insect. Soc. 39: $313-324$

Roisin Y. 1996. Castes in humivorous and litter-dwelling neotropical nasute termites (Isoptera, Termitidae). Insect. Soc. 43: 375-389

Roisin Y. 2000. Diversity and evolution of caste patterns. In: Termites: Evolution, Sociality, Symbioses, Ecology (Abe T., Bignell D.E. and Higashi M. Eds.), Dordrecht, the Netherlands: Kluwer Academic Publishers. pp 95-119

Roisin Y. 2001. Caste sex ratios, sex linkage, and reproductive strategies in termites. Insect. Soc. 48: $224-230$

Roisin Y. and Lenz M. 1999. Caste developmental pathways in colonies of Coptotermes 
lacteus (Froggatt) headed by primary reproductives (Isoptera, Rhinotermitidae). Insect. Soc. 46: 273-280

Roisin Y. and Pasteels J.M. 1987. Caste developmental potentialities in the termite Nasutitermes novarumhebridarum. Entomol. Exp. Appl. 44: 277-287

Ronquist F. and Huelsenbeck J.P. 2003. MrBayes 3: Bayesian phylogenetic inference under mixed models. Bioinformatics 19: 1572-1574

Stewart A.D. and Zalucki M.P. 2006. Developmental pathways in Microcerotermes turneri (Termitidae: Termitinae): Biometric descriptors of worker caste and instar. Sociobiology 48: $727-740$

Tamura K., Peterson D., Peterson N., Stecher G., Nei M. and Kumar S. 2011. MEGA5: Molecular evolutionary genetics analysis using maximum likelihood, evolutionary distance, and maximum parsimony methods. Mol. Biol. Evol. 28: 2731-2739

Thorne B.L. 1997. Evolution of eusociality in termites. Annu. Rev. Ecol. Syst. 28: 27-54

Trivers R.L. and Hare H. 1976. Haplodiploidy and the evolution of the social insects. Science 191: $249-263$ 
Fig. 1. Sex of soldier caste according to sexual size dimorphism.

Fig. 2. Phylogenetic tree reconstructed using Maximum Likelihood and generated using COII, $12 \mathrm{~S}$ and $28 \mathrm{~S}$ sequence data from termites. A. Ancestral reconstruction of single sex soldier using a likelihood method with the AsymmMk models; B. Ancestral reconstruction of sexual size dimorphism using a likelihood method with the AsymmMk models. All analyses were performed using Mesquite (Maddison and Maddison 2010). Ancestral state reconstruction of (A) single-sex soldiers and (B) SSD using the parsimony and likelihood methods with the Mk1 model provided similar results.

Fig. 3. Bayesian phylogenetic tree generated using COII, $12 \mathrm{~S}$ and $28 \mathrm{~S}$ sequence data from termites. A. Ancestral reconstruction of single sex soldier using a likelihood method with the AsymmMk models; B. Ancestral reconstruction of sexual size dimorphism using a likelihood method with the AsymmMk models. All analyses were performed using Mesquite (Maddison and Maddison 2010). Ancestral state reconstruction of (A) single-sex soldiers and (B) SSD using the parsimony and likelihood methods with the Mk1 model provided similar results. 

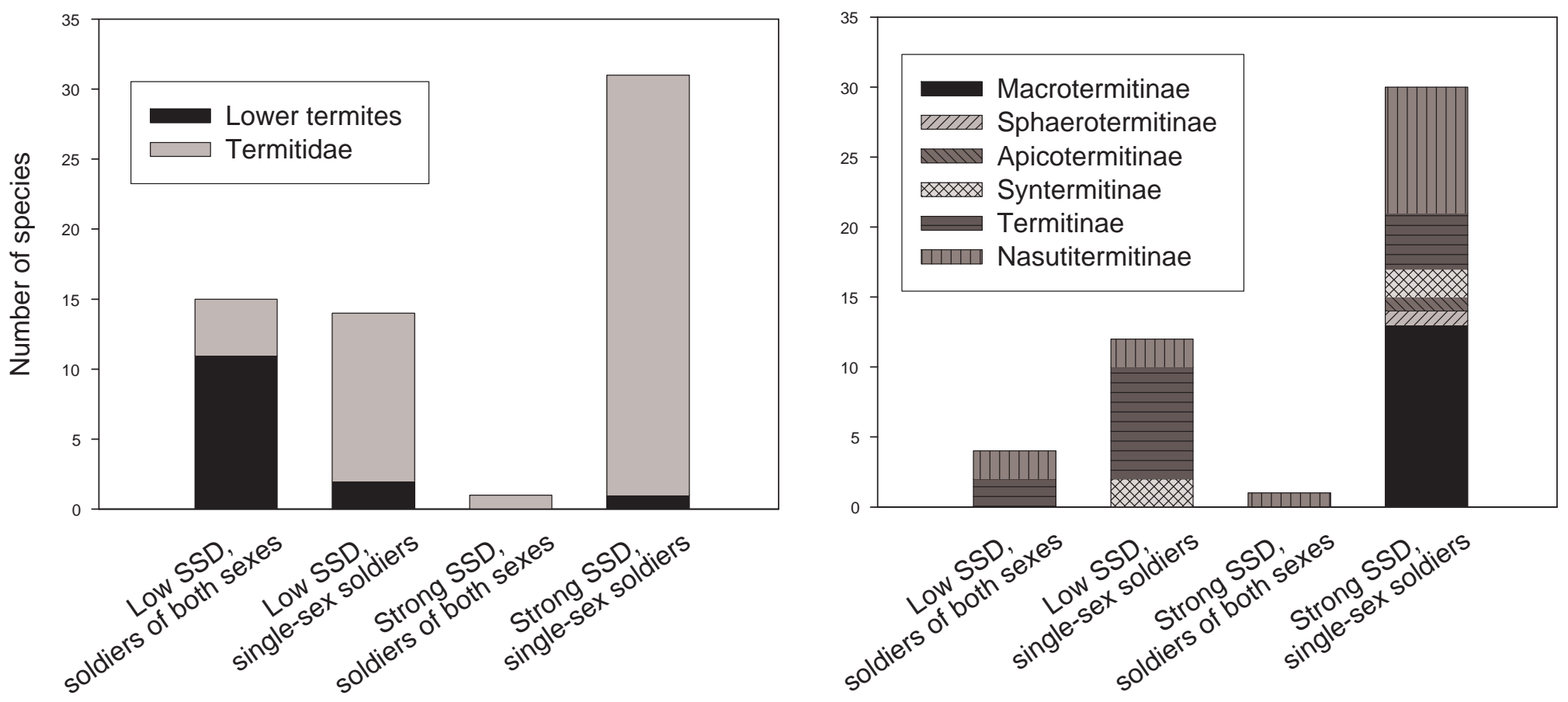
Soldiers of both sexes

Single-sex soldiers

a
Prorhinotermes

Termitogeton planus Macrotermes

Ancistrotermes crucifer Odontotermes

Protermes minutus

Pseudacanthotermes

Microtermes

Acanthotermes acanthothorax

Microcerotermes parvus

Labiotermes labralis

Syntermes

Embiratermes

Orthotermes depressifrons

Nasutitermes arborum

Trinevitermes

Hospitalitermes

Coatitermes

Subulitermes

Velocitermes

Leptomyxotermes doriae

Fulleritermes

Cubitermes

Thoracotermes

Amitermes evuncifer

Termes hospes

Pericapritermes

Labidotermes

Sphaerotermes sphaerothorax Coptotermes formosanus

Coptotermes lacteus

Reticulitermes kanmonensis

Reticulitermes speratus

Reticulitermes virginicus

Reticulitermes flavipes Neotermes

Cryptotermes domesticus

Cryptotermes secundus

Hodotermopsis sjoestedti

Hodotermes mossambicus

Zootermopsis angusticollis

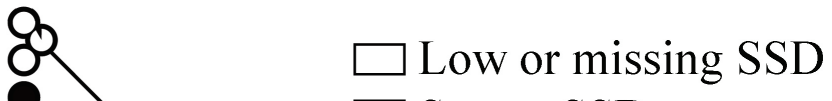

- Strong SSD 

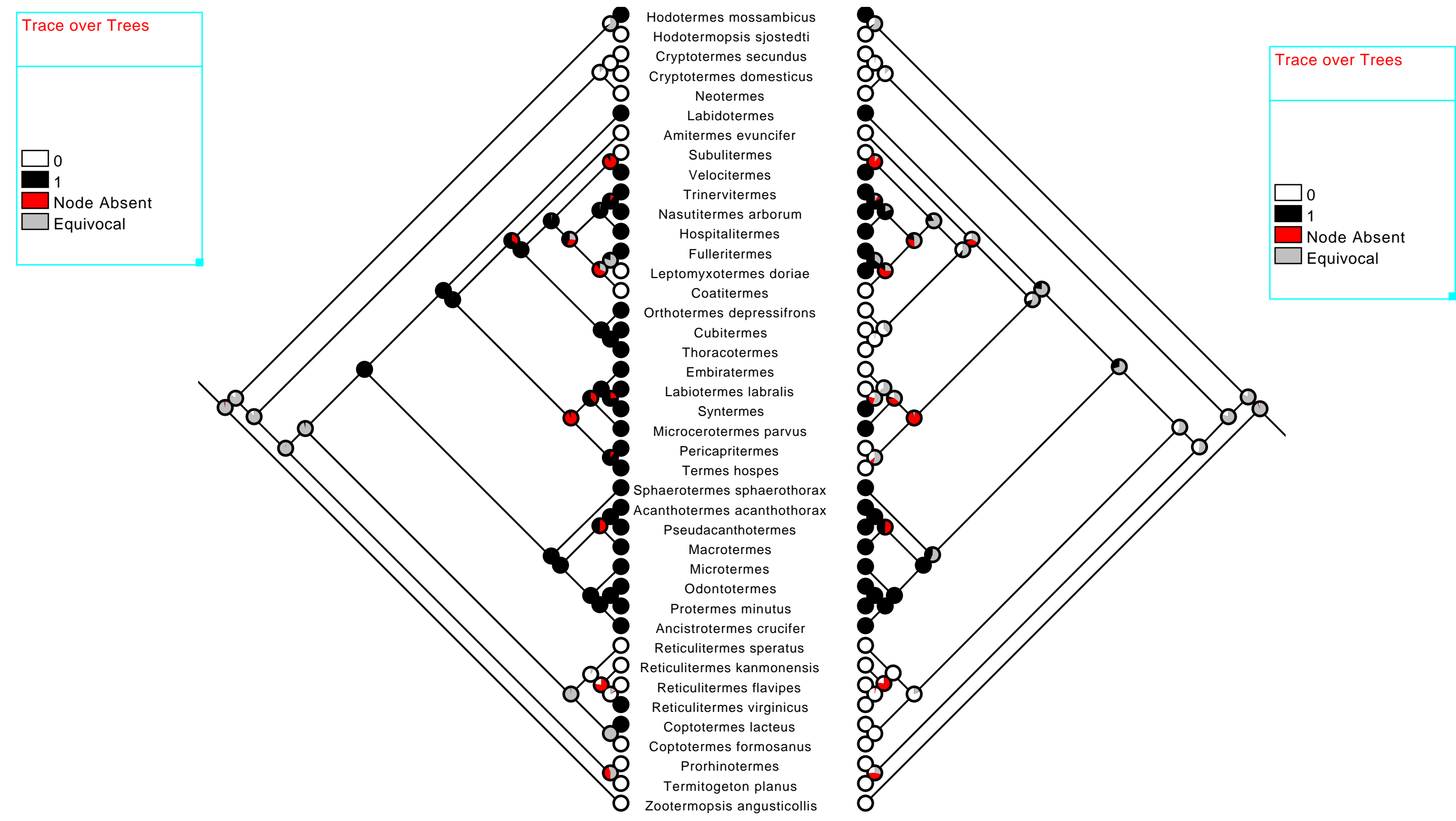